\title{
X-Ray Grating Interferometry - Applications in Metrology and Wave Front Sensing
}

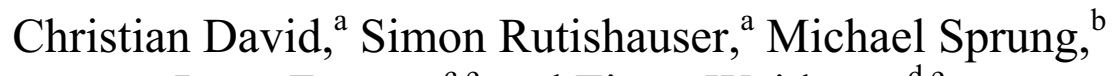 \\ Irene Zanette, ${ }^{\mathrm{c}, \mathrm{e}}$ and Timm Weitkamp ${ }^{\mathrm{d}, \mathrm{e}}$ \\ ${ }^{a}$ Paul Scherrer Institut, 5232 Villigen, Switzerland \\ ${ }^{b}$ Deutsches Elektronen Synchrotron, 22607 Hamburg, Germany \\ ${ }^{c}$ Technische Universität München, 85748 Garching, Germany \\ ${ }^{d}$ Synchrotron Soleil, 91192 Gif-sur-Yvette, France \\ ${ }^{e}$ European Synchrotron Radiation Facility, 38000 Grenoble, France
}

\begin{abstract}
X-ray grating interferometry was first developed in context with x-ray phase contrast imaging. Here we focus on applications where 1- and 2-dimensional grating interferometers are used to measure the distortions of $\mathrm{x}$-ray wave fronts with extreme sensitivity. This allows for the in-situ and at wavelength characterization on x-ray optics such as mirrors and refractive lenses. Angular sensitivities down to the 10 nrad range can be obtained. We point out some specific concerns when using the technique in context with metrology. A precise rotational alignment is essential for accurate, absolute measurements of the local wavefront radius of curvature. Moreover, in many cases no flat-field image can be recorded, therefore the gratings need to fulfill very stringent requirements with respect to distortions.
\end{abstract}

Keywords: x-ray optics, wave front, metrology, free-electron lasers, moiré

PACS: $42.15 . \mathrm{Dp} ;$ 42.30.Rx; 42.55.Vc; 41.50. $+\mathrm{h} ; 0$ 07.85.Fv

\section{INTRODUCTION}

About a decade ago, first work on x-ray grating interferometry was started $[1,2]$ with the goal to visualize the $\mathrm{x}$-ray phase shift induced by a sample and to measure it in a quantitative way. The method is based on detecting refraction of $x$-rays on phase gradients in the sample, which has led to the frequently used term "differential phase contrast (DPC) imaging". More generally speaking, a grating interferometer is a very sensitive tool for the detection of x-ray wavefront distortions, which makes it well suited for using it for wavefront metrology, in particular to investigate the effect of optical elements used in x-ray beam lines, such as crystal monochromators, multilayer or grazing incidence mirrors.

Usually, the quality of such optical elements is assessed by methods such as long trace profilers (LTPs) or visible light interferometers. These techniques have been developed to a very high degree of perfection. Shape errors on the order of nanometers and slope errors down to the 100 nrad level are detectable, even on very long mirror substrates [3]. However, at the same time, the quality demands for state-of-the-art $\mathrm{x}$ ray optics have reached a level where even narrower fabrication tolerances need to be fulfilled for ultimate performance. This means that the optics metrology techniques form a bottle neck for the further improvement of $\mathrm{x}$-ray optical instrumentation.

\footnotetext{
International Workshop on X-ray and Neutron Phase Imaging with Gratings

AIP Conf. Proc. 1466, 23-28 (2012); doi: 10.1063/1.4742264

(C) 2012 American Institute of Physics 978-0-7354-1072-5/\$30.00
} 
In addition to the technological limits of metrology tools, a more fundamental problem arises when using the above-mentioned techniques. As they are performed off-line, even a perfect measurement would not include distortions induced by the transfer from the metrology site, by the mounting at the beam line, and by imperfections of the alignment. Moreover, thermal deformations caused by the heat load of the x-ray beam are not detected. Therefore, an in-situ, at-wavelength method provides substantial advantages. In addition, it could be used as a feed-back to reduce distortions, e.g. by optimizing the shape though actuators in an adaptive mirror.

\section{MOIRE FRINGE ANALYSIS FOR WAVEFRONT SENSING}

When using x-ray grating interferometry for phase contrast imaging, there are two fundamental modes of operation. Either the refraction in the sample is determined by recording a phase stepping series [4], or by analyzing the deformation of moiré fringes [5]. The phase-stepping mode requires the acquisition of multiple images but yields high spatial resolution, and the data are easy to process. The moiré mode, however, can provide differential wavefront information from single images. This can be essential when working on pulsed sources like an X-FEL, and we consequently used the moiré mode for the measurements presented in this paper.

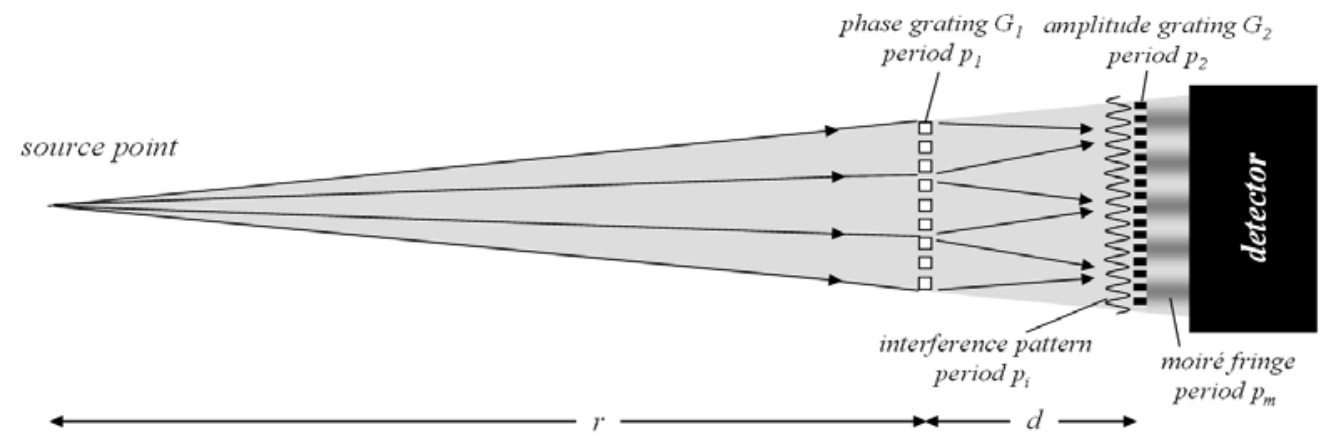

FIGURE 1. Schematic of a grating interferometer consisting of a phase shifting grating $\mathrm{G}_{1}$ with pitch $p_{1}$, placed at a distance $r$ from the source point, and an absorbing grating $\mathrm{G}_{2}$ with pitch $p_{2}$ placed at a distance $d$ downstream of $\mathrm{G}_{1}$.

The grating interferometer discussed here consists of a $\pi$-phase shifting grating $\mathrm{G}_{1}$, which is illuminated by a source at a distance $r$ and generates an interference pattern with a period $p_{i}$ in a plane at a distance $d$ downstream of $\mathrm{G}_{1}$ (see Fig. 1). Due to the Talbot effect, the contrast of the fringes depends on $d$; it can even drop to zero for distances where a self-image of the phase grating $\mathrm{G}_{1}$ is created. Maximum contrast can be observed for odd fractional Talbot distances [2]. The fringe pattern is analyzed by a second grating $\mathrm{G}_{2}$ with absorbing lines and a pitch $p_{2}$. The transmitted pattern detected by the camera shows moire fringes with a period $p_{m}$. Let us consider the case that the gratings are perfectly aligned with respect to their rotation around the optical axis. In this case, $p_{i}$ and $p_{m}$ are given by:

$$
p_{i}=\frac{p_{1}(r+d)}{2 r} \quad \text { and } \quad p_{m}=\left|\frac{p_{i} \cdot p_{2}}{p_{i}-p_{2}}\right|
$$


For an interferometer with $p_{\mathrm{i}}=p_{2}, p_{\mathrm{m}} \rightarrow \infty$, this means that there will be no moiré fringes when $G_{1}$ and $G_{2}$ are perfectly aligned. This requires that the quantity $r_{0}=d \cdot p_{1} /\left(2 p_{2}-p_{1}\right)$, which we shall refer to as the interferometer radius, is matched to the radius of curvature $r$ of the incoming wave. For a plane wave (i.e. $r \rightarrow \infty$ ), this is the case for $p_{2}=p_{1} / 2$.

When there is a rotational misalignment of the gratings by an angle $2 \beta$, a matched interferometer will give moiré fringes that are oriented perpendicular to the grating lines, as shown in Fig. 2a. When the interferometer radius $r_{0} \neq r$, a rotational misalignment will lead to a tilt angle $\theta_{\mathrm{m}}$ moiré fringes, see Fig. $2 \mathrm{~b}$. This angle $\theta_{\mathrm{m}}$ is can be used to measure the wave front radius $r$ using the following equation:

$$
\frac{1}{r}=\frac{1}{r_{0}}-\frac{2 \beta \cdot \tan \theta_{m}}{d}=\frac{2 p_{2} / p_{1}-2 \beta \cdot \tan \theta_{m}-1}{d}
$$

A detailed derivation of the related formulas for the moiré fringe orientation can be found in ref. [6].

a

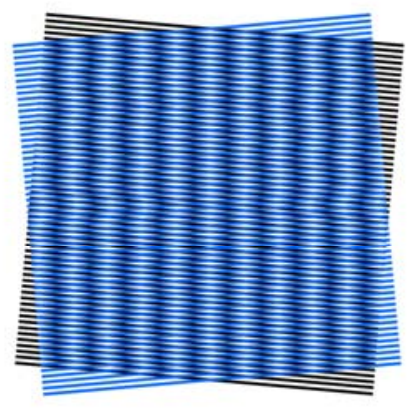

b

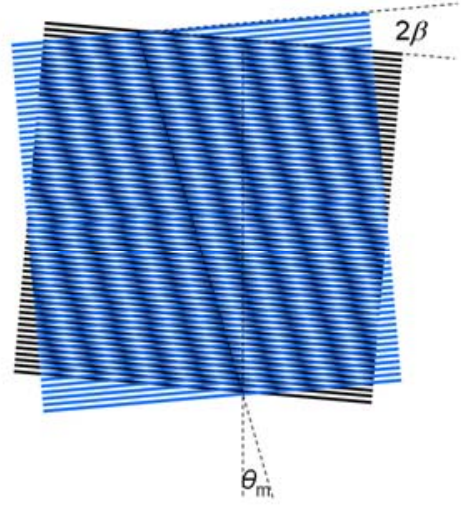

FIGURE 2. The moiré fringes generated in grating interferometry by rotation of the gratings by $2 \beta$ :

a) the matched case, when $r_{0}=r$, and b) for $r_{0} \neq r$.

\section{Absolute measurements of the wave front radius}

Based on eq. 2, the angle of a moiré fringe pattern can be directly used to determine a wave front radius. However, a rotational misalignment of the image detector also affects the apparent fringe angle. In order to separate the two effects, it is therefore crucial to measure the camera alignment with high precision. This can be done by taking a series of images while scanning the rotation angle $2 \beta$ between $\mathrm{G}_{1}$ and $\mathrm{G}_{2}$. Fig. 3 shows five experimental data of such a series obtained at beamline P10 of the PETRA3 source in Hamburg, Germany. The gratings were mounted with horizontal orientation of the grating lines. For analysis of the fringe angle we apply a 2dimensional Fourier transform. The bright spot in the center (i.e. at the origin) represents the DC offset, while the other two spots represent the fundamental frequency and orientation of the moiré fringes. By scanning the angle in fine steps and applying an automated peak detection algorithm, we can plot the graph shown in Fig. 4a. The data points do not lie on straight lines, because in this experiment only $\mathrm{G}_{2}$ was 
(a)
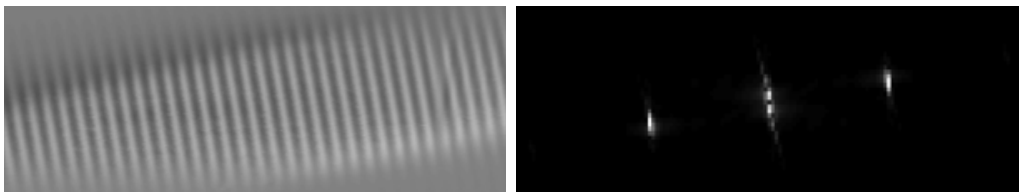

(b)
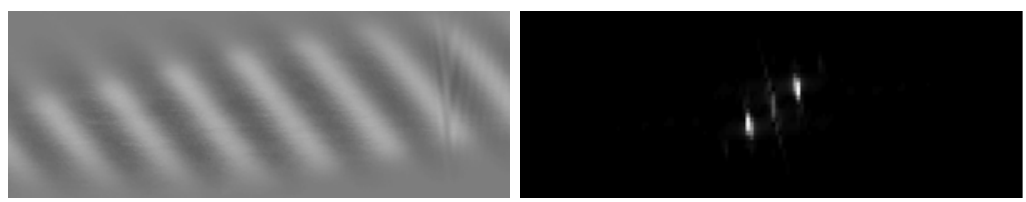

(c)
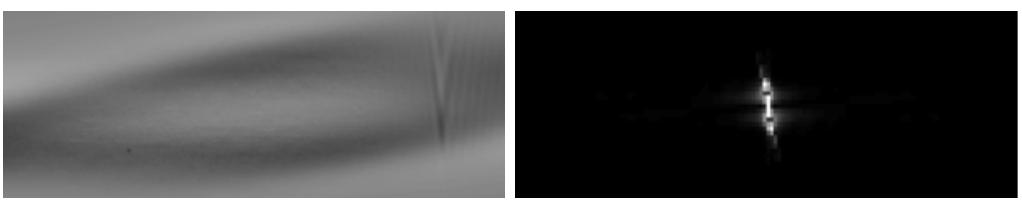

(d)
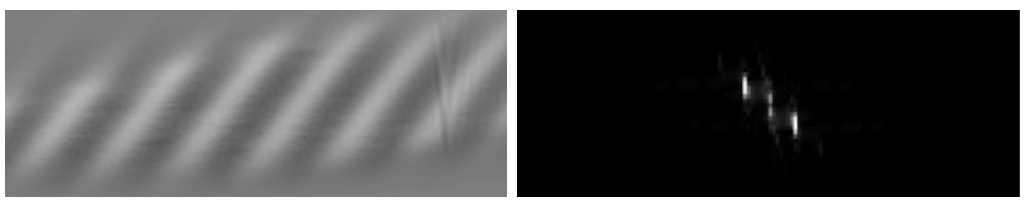

(e)
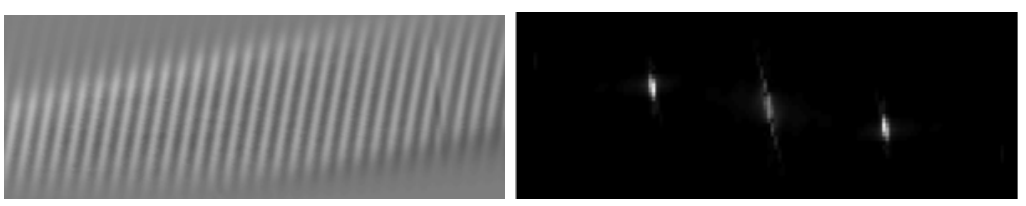

FIGURE 3. Moiré fringe patterns for different angles $\beta$ between $\mathrm{G}_{1}$ and $\mathrm{G}_{2}$ (left) and the corresponding 2D Fourier transform (right). Photon energy: $24 \mathrm{keV}$, interferometer parameters: $\mathrm{p}_{1}=$ $3.981 \mu \mathrm{m}, \mathrm{p}_{2}=2.000 \mu \mathrm{m}, \mathrm{d}=423.8 \mathrm{~mm}, \mathrm{r}_{0}=88 \mathrm{~m}$. The images (c) display the situation where $\beta \approx 0$.
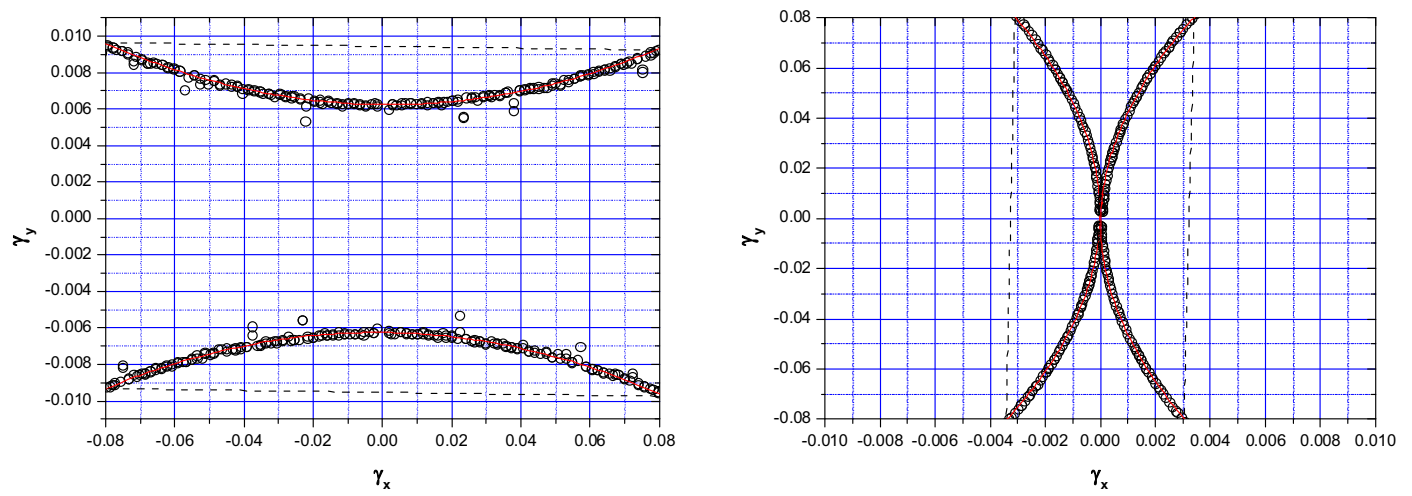

FIGURE 4. Peak positions in the Fourier transformed moiré fringe images for a dense scan of the grating rotation $\beta$. The axes indicate the spatial frequencies $\gamma_{\mathrm{x}}$ and $\gamma_{\mathrm{y}}$ in units of $p_{2} / p_{\mathrm{m}}$. The symbols represent individual measurements, the red line is a numerical fit. Left: grating lines oriented in horizontal direction. The gap between the curves stems from a mismatch between the vertical wave front radius $r$ and the interferometer radius $r_{0}$. Right: same measurement with the grating lines oriented in vertical direction. Here the interferometer is well matched $\left(r \approx r_{0}\right)$.

rotated. Moreover, the curves do not touch at the origin, which indicates, that the wavefront radius in vertical direction $r_{\mathrm{y}}$ differs significantly from $r_{0}$. The red curves are fits of an analytical expression derived by Wang et al. [7]. This yields the camera misalignment of $1.6 \mathrm{mrad}$ (indicated by the slight slope of the dashed lines), and a 
vertical wave front radius of $-281 \mathrm{~m}$. A negative radius corresponds to a converging beam, focused to a point $281 \mathrm{~m}$ downstream of the interferometer. This surprising finding can be attributed to a focusing of the $\mathrm{Si}<111>$ double crystal monochromator. Applying the same analysis to data taken with the grating lines oriented in perpendicular direction gives the data shown in Fig. 4b. The horizontal wave front radius is $89.5 \mathrm{~m}$. The latter value is in excellent agreement with the source distance of $88 \mathrm{~m}$. The discrepancy between the two directions is due to the fact that the monochromator is vertically deflecting under a shallow Bragg angle, which significantly increases its effect on the wavefront radius in vertical direction.

\section{Optics metrology}

The technique can also be applied to measure fringe angles (and thus the wavefront radius) in a spatially resolved way, i.e. for different regions of an image. Such a wave front analysis of a beam reflected by multilayer mirror was first demonstrated by Weitkamp et al. [8]. Slope errors on the order of some tens of nanoradians were detected. The method can also be expanded to two-dimensional grating interferometers [9]. An example is shown in Fig. 5, which displays the wave front deformations caused by a refractive x-ray lens made from beryllium [10]. Although the focusing effect of this single lens is weak (focal length: $776 \mathrm{~m}$ ), it causes a clear clockwise rotation of the two-dimensional fringe pattern in the central, round aperture of the lens. A more detailed analysis of these experiments is reported elsewhere [11].

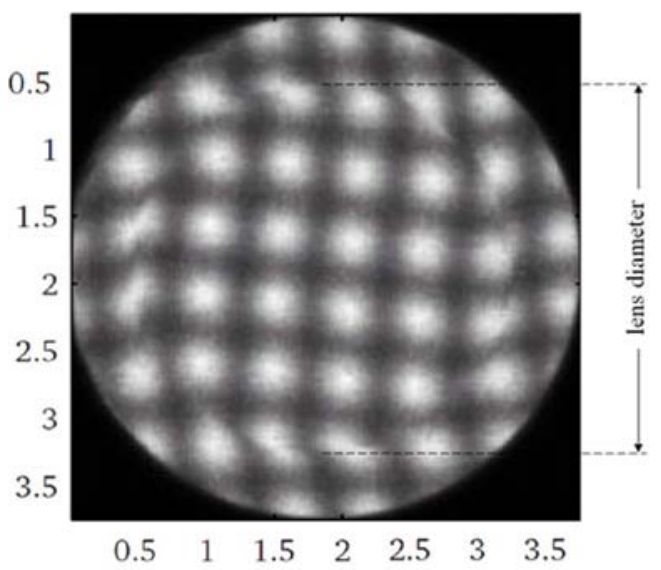

FIGURE 5. Moiré fringe pattern recorded at beam line ID19 of the ESRF, Grenoble, using a 2dimensional grating interferometer. The axes show the dimensions of the image in millimeters. The central region is distorted by a beryllium refractive lens with an aperture of about $2.5 \mathrm{~mm}$. Photon energy: $23 \mathrm{keV}$, interferometer parameters: $\mathrm{p}_{1}=4.785 \mu \mathrm{m}, \mathrm{p}_{2}=2.400 \mu \mathrm{m}, \mathrm{d}=479 \mathrm{~mm}, \mathrm{r}_{0}=153 \mathrm{~m}$.

\section{Grating accuracy requirements}

The use of x-ray grating interferometry for imaging applications generally involves the acquisition of a flat-field image, i.e., a data set taken with the sample removed from the beam. In DPC imaging, a flat-field correction does not only cancel out inhomogeneities in the illumination or the detector response. It also provides a phase 
flat-field, which helps remove effects by distortions of the incoming wave front, imperfect alignment, and distortions of the gratings. The case of the refractive lens shown in Fig. 5 is one of the few cases in optics metrology where recording of a flatfield is possible. Generally, one has to rely on the quality of the illumination and the grating. The latter is essentially given by the placement accuracy of the lithography tools used for grating fabrication. Assuming a grating distance $d$ of $500 \mathrm{~mm}$ (typical of high-resolution interferometers at synchrotrons), a placement error of $5 \mathrm{~nm}$ in the grating lines corresponds to an error of the wave-front angle measurement of $10 \mathrm{nrad}$.

Even state-of-the-art electron beam writers cannot reliably provide such accuracy over large areas (several millimeters) due to stitching errors at the write field boundaries. We apply an electron writing technique in continuous path control mode [12], which enables the exposure of several millimeter large gratings without the need for write field stitching. A proper match of the grating periods to the address grid of the lithography tool can help to avoid commensurability problems [13].

\section{ACKNOWLEDGMENTS}

We wish to thank the staff of the Laboratory for Micro- and Nanotechnology and of the beamlines P10 and ID19 for their support. Some of the $\mathrm{G}_{2}$ gratings used in these experiments were supplied by the Karlsruhe Institute of Technology, for which the support of the Karlsruhe Nano Micro Facility (KNMF) is acknowledged. T.W. acknowledges support from RTRA "Digiteo" and "Triangle de la Physique.

\section{REFERENCES}

1. C. David, B. Nöhammer, H.H. Solak, E. Ziegler, Appl. Phys. Lett, 81 (2002) pp. 3287-3289

2. A. Momose, S. Kawamoto, I. Koyama, Y. Hamaishi, K. Takai, Y. Suzuki, Jpn. J. Appl. Phys., 42 (2003) L866-L868

3 F. Siewert, T. Noll, T. Schlegel, T. Zeschke, H. Lammert, AIP Conference Proceedings 705 (2004) pp. $847-850$

4. T. Weitkamp, A. Diaz, C. David, F. Pfeiffer, M. Stampanoni, P. Cloetens, and E. Ziegler, Opt. Express 13 (2005) pp. 6296-6304

5. A. Momose, W. Yashiro, H. Maikusa, Y. Takeda, Opt. Express 17, (2009) pp. 12540-12545

6. T. Weitkamp, A. Diaz, B. Nöhammer, F. Pfeiffer, M. Stampanoni, E. Ziegler, and C. David Proc. SPIE 5533 (2004) pp. 140-144

7. H. Wang, K. Sawhney, S. Berujon, E. Ziegler, S. Rutishauser, C. David, Opt. Express 19 (2011) pp. 16550-16559

8 T. Weitkamp, B. Nöhammer, A. Diaz, C. David, E. Ziegler., Appl. Phys. Lett. 86 (2005) 054101

9. I. Zanette, T. Weitkamp, T. Donath, S. Rutishauser, and C. David, Phys. Rev. Lett. 105 (2010) 248102

10.B. Lengeler, C.G Schroer, M. Kuhlmann, B. Benner, T.F. Günzler, O. Kurapova, F. Zontone, A. Snigirev, I. Snigireva J. Phys. D: Appl. Phys. 38 (2005) pp. A218-A222

11. S. Rutishauser, I. Zanette, T. Weitkamp, T. Donath, and C. David. Appl. Phys. Lett. 98 (2011) 171107-171109

12.C. David and D. Hambach, Microelectron. Eng. 46 (1999) pp. 219-222

13.B. Schnabel, E.B. Kley, Microelectron. Eng. $57-58$ (2001) pp. 327-333 